This is an Open Access article, distributed under the terms of the Creative Commons Attribution licence (http://creativecommons.org/licenses/by/4.0/), which permits unrestricted re-use, distribution, and reproduction in any medium, provided the original work is properly cited.

doi:10.1017/jfm.2017.24

\title{
Very-large-scale motions in rough-bed open-channel flow
}

\author{
S. M. Cameron ${ }^{1} \dagger$, V. I. Nikora ${ }^{1}$ and M. T. Stewart ${ }^{1}$ \\ ${ }^{1}$ School of Engineering, University of Aberdeen, Aberdeen AB24 3UE, UK \\ (Received 9 August 2016; revised 5 January 2017; accepted 7 January 2017; \\ first published online 9 February 2017)
}

Long-duration particle image velocimetry measurements in rough-bed open-channel flows $(\mathrm{OCFs})$ reveal that the pre-multiplied spectra of the streamwise velocity have a bimodal distribution due to the presence of large- and very-large-scale motions (LSMs and VLSMs, respectively). The existence of VLSMs in boundary layers, pipes and closed channels has been acknowledged for some time, but strong supporting evidence for their presence in OCF has been lacking. The data reported in this paper fill this gap. Length scales of the LSMs and VLSMs in OCF exhibit different scaling properties; whereas the streamwise length of the LSM scales with the flow depth, the VLSM streamwise length does not scale purely with flow depth and may additionally depend on other scales such as the channel width, roughness height or viscous length. The transverse extent of the LSMs was found to increase with increasing elevation, but the VLSM transverse scale is anchored around two flow depths. The origin and nature of LSMs and VLSMs are still to be resolved, but differences in their scaling suggest that VLSMs in rough-bed OCFs form independently rather than as a spatial alignment of LSMs.

Key words: turbulent boundary layers, turbulent flows

\section{Introduction}

Kim \& Adrian (1999) discovered a bimodal distribution in the streamwise velocity pre-multiplied spectrum of pipe flow and described the two apparent scales as largeand very-large-scale motions (LSMs and VLSMs, respectively). They proposed that VLSMs may result from a streamwise alignment of LSMs, which themselves might represent a collection of smaller hairpin-shaped vortices. Subsequently, VLSMs have been discovered in other flow types, although their origin remains unclear, with other authors proposing that VLSMs could form independently due to large-scale instability (e.g. Hwang \& Cossu 2010). In the case of boundary layers, Hutchins \& Marusic (2007) introduced the term 'superstructures' although it is possible that these have the same origin as VLSMs, albeit different characteristics due to fundamental differences in the boundary conditions of pipe, closed-channel and boundary layer flows. Comparing pre-multiplied spectra across these three flow types, Monty et al.

$\dagger$ Email address for correspondence: s.cameron@abdn.ac.uk 
(2009) found a remarkable resemblance between pipe and closed-channel flows; boundary layers in comparison exhibit superstructures in the logarithmic layer, being significantly suppressed in the outer layer.

The presence of VLSMs in open-channel flow (OCF) has not been firmly established yet, although such a test would be important, as a number of specific OCF features (e.g. free water surface) make them distinctly different from pipes, closed channels and boundary layers. Indeed, if we consider boundary layers as 'external' flows and pipes and closed channels as 'internal' flows, then the OCF may be viewed as a flow occupying an intermediate position between them, exhibiting features of both external and internal flow types. This feature makes OCF a particularly interesting object to study in relation to VLSM occurrence and dynamics.

Most studies of VLSMs and superstructures in pipes, closed channels and boundary layers have been undertaken with smooth-wall conditions. Although smooth-wall conditions are also relevant to OCFs, most practical situations involve rough-bed OCFs and thus their study is of the utmost importance. There are many engineering challenges - such as the assessment of bed stability, sediment transport, mixing and hydraulic resistance in rivers and canals - that require the advanced understanding of rough-bed OCF turbulence. Therefore, the focus of this study is on rough-bed OCF.

The current knowledge on turbulent motions in OCFs mainly relate to scales that do not exceed 5-8 flow depths (e.g. Nezu \& Nakagawa 1993; Roy et al. 2004; Nezu 2005; Franca \& Brocchini 2015). Nevertheless, there are also indications of much larger-scale motions in OCFs. As an example, Grinvald \& Nikora (1988) performed long-term (up to 4 hours) velocity measurements in a number of lowland rivers and found that velocity spectra exhibit low-frequency peaks corresponding to wavelengths, via Taylor's 'frozen' turbulence hypothesis, up to several channel widths or up to a hundred flow depths. These low-frequency velocity fluctuations have been interpreted in Grinvald \& Nikora (1988) as a signature of 'horizontal' turbulence. They showed that these motions modulate depth-scale motions and near-bed turbulence. In light of the recent discovery of VLSMs in other flow types, the revealed low-frequency spectral peaks could also reflect very long meandering 'strips' of low and high momentum. Field data of Franca \& Lemmin (2005) support this later scenario. There are also a number of laboratory studies (e.g. Nezu 2005) that indicate the presence of long rotational motions resembling VLSMs in pipes, closed channels and boundary layers. A fairly comprehensive review of long rotational motions that are occasionally reported for OCFs can be found in Adrian \& Marusic (2012). They stated that the 'close relationship between meandering VLSMs of turbulence structure research and the long cellular motions of open-channel flow research is impossible to ignore. It seems likely, in fact, that they are one and the same' (Adrian \& Marusic 2012, pp. 451-460). The next step then would be to undertake a high-precision study with measurement resolution and extent that are sufficient for confident identification and quantification of VLSMs in OCFs. If VLSMs are confirmed in OCF, then the assessment of their roles in processes occurring in river flows and important for hydraulic engineering may lead to significant scientific advances and much improved engineering models.

Thus, the objective of this study is to identify and quantify VLSMs in rough-bed OCF using long-duration ( 2 hours) particle image velocimetry (PIV) measurements. The focus is on OCFs at low and intermediate submergences, which remain the least studied flow configurations in spite of their ubiquitous occurrence in nature and engineering. Section 2 outlines the experimental facility, instrumentation and procedures. Section 3 summarises the background hydraulic conditions, including bulk statistics, velocity correlations, spectra and turbulence scales. Finally, the main findings are briefly outlined in $\S 4$. 


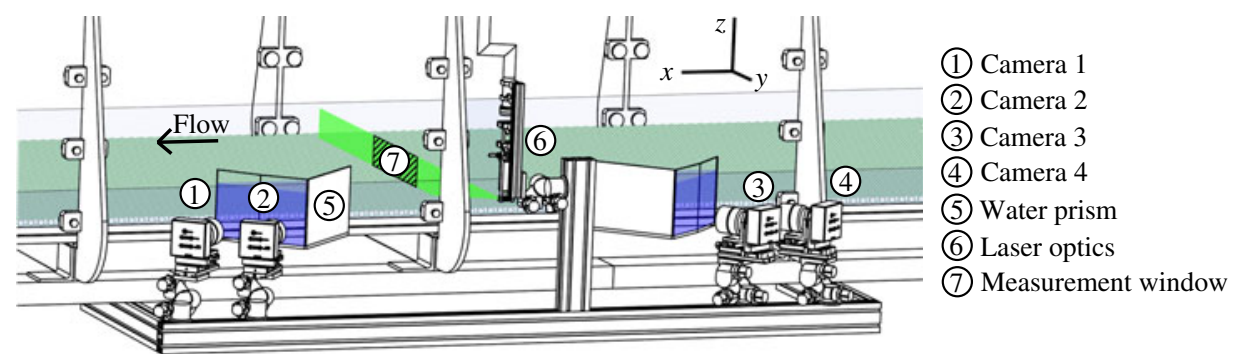

Figure 1. (Colour online) Measurement section of the Aberdeen Open-Channel Facility.

\section{Experimental set-up}

\subsection{Open-channel flume and flow configurations}

Experiments were conducted in the Aberdeen Open-Channel Facility (AOCF). The facility consists of a $1.18 \mathrm{~m}$ wide and $18 \mathrm{~m}$ long recirculating open-channel flume and a motorised instrumental carriage that houses a PIV system and supporting instrumentation (figure 1). A vertical slat weir regulates the backwater curve, which we measure by profiling the water surface with an ultrasonic displacement sensor attached to the instrumental carriage. The entire bed of the flume and part of the entrance tank (19.2 m total length) was covered for these experiments with a single layer of $16 \mathrm{~mm}$ diameter $(D$, figure $2 a)$ glass spheres. The spheres were precisely aligned in a hexagonally close-packed arrangement with the help of perforated stainless-steel plates. The installed plates were levelled using shims to within $\pm 0.25 \mathrm{~mm}$. Experiments were conducted with relative submergences $(H / D)$ between 1.9 and 7.5 (table 1) and constant shear velocity $\left(u_{*}=0.042 \mathrm{~m} \mathrm{~s}^{-1}\right)$. The roughness Reynolds number $D^{+}=D u_{*} / v$ was 605 for all experiments, indicating fully rough flow conditions, as justified in Cameron, Nikora \& Coleman (2008). The friction Reynolds number $H^{+}=H u_{*} / v$ varied between 1140 and 4540, being similar to the experiments of Kim \& Adrian (1999) in pipes (1058-3175) and the measurements of Hutchins \& Marusic (2007) in boundary layers (1020-19960). The Froude number was well below 1.0, securing a flat water surface without visible surface waves; measured standard deviations of water surface level varied within $0.28-0.45 \mathrm{~mm}$. The flows were uniform, with water surface slope deviating from the bed surface slope by a maximum of $2.5 \times 10^{-5}$, and steady to within a flow rate standard deviation of $0.0002 \mathrm{~m}^{3} \mathrm{~s}^{-1}$.

\subsection{Stereoscopic particle image velocimetry}

A four-camera stereoscopic PIV system was used to measure all three velocity components in a wall-normal transverse plane (figure 1). The measurement plane covered the full flow depth, extended $330 \mathrm{~mm}$ across the flow and was positioned $12.7 \mathrm{~m}(>100 \mathrm{H})$ downstream from the flume entrance. Under equivalent flow conditions, Stewart (2014) demonstrated that this distance was sufficient for fully developed conditions to exist at the test section. The four cameras (Dalsa 4M60, $2352 \times 1728$ pixels, $7.4 \mu \mathrm{m}$ pixel pitch, $60 \%$ effective fill factor, 8 bits per pixel, Nikon $60 \mathrm{~mm}$ lens at f/16 aperture) were positioned symmetrically with respect to the light sheet at angles of $\pm 45^{\circ}$ and $\pm 38^{\circ}$ for cameras 1 and 4 and cameras 2 and 3 , respectively (figure 1). The laser (Oxford Lasers dual-cavity Nd:YAG, $532 \mathrm{~nm}$, $100 \mathrm{~mJ}$ pulse $\mathrm{s}^{-1}$ ) output was formed into a $2.4 \mathrm{~mm}$ thick sheet using a series of 
(a)

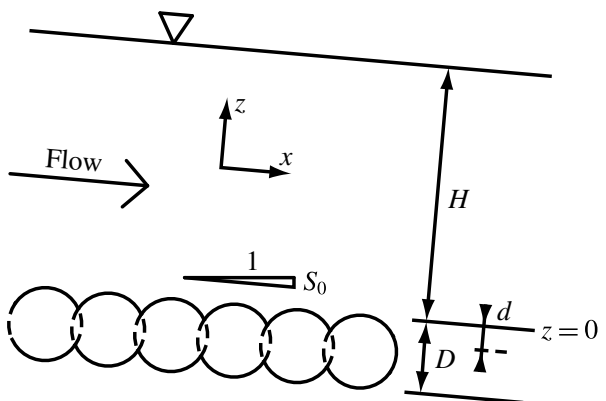

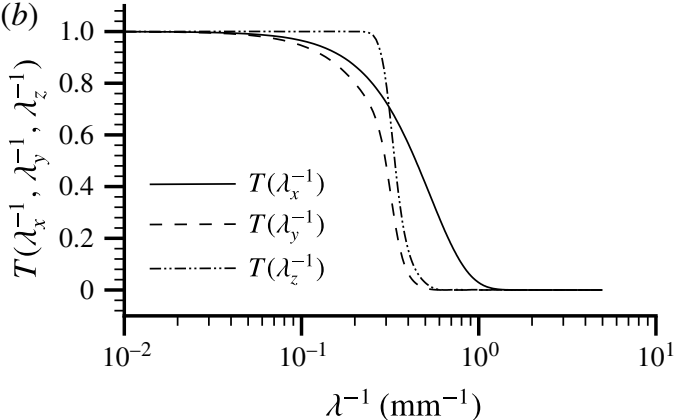

FIgURE 2. (a) Definition of flow depth $(H)$, particle diameter $(D)$, bed surface slope $\left(S_{0}\right)$ and zero-plane displacement $(d)$ for the log law; $(b)$ transfer function of the PIV measurements.

\begin{tabular}{lrcccccccccc}
\hline Run & $\begin{array}{c}H \\
(\mathrm{~mm})\end{array}$ & $\begin{array}{c}Q \\
\left(\mathrm{~m}^{3} \mathrm{~s}^{-1}\right)\end{array}$ & $\begin{array}{c}U \\
\left(\mathrm{~m} \mathrm{~s}^{-1}\right)\end{array}$ & $S_{0}$ & $\begin{array}{c}u_{*} \\
\left(\mathrm{~m} \mathrm{~s}^{-1}\right)\end{array}$ & $R$ & $H^{+}$ & $D^{+}$ & $H / D$ & $B / H$ & $F r$ \\
H030 & 30.1 & 0.0153 & 0.431 & 0.00600 & 0.042 & 11700 & 1140 & 605 & 1.9 & 39.2 & 0.79 \\
H050 & 50.3 & 0.0275 & 0.463 & 0.00360 & 0.042 & 21000 & 1900 & 605 & 3.1 & 23.5 & 0.66 \\
H070 & 70.5 & 0.0404 & 0.486 & 0.00257 & 0.042 & 30800 & 2670 & 605 & 4.4 & 16.7 & 0.58 \\
H095 & 94.9 & 0.0569 & 0.508 & 0.00189 & 0.042 & 43400 & 3590 & 605 & 5.9 & 12.4 & 0.53 \\
H120 & 120.1 & 0.0745 & 0.526 & 0.00150 & 0.042 & 56900 & 4540 & 605 & 7.5 & 9.8 & 0.48
\end{tabular}

TABLE 1. Flow conditions for the experiments: $H$ is flow depth above the roughness tops, $B$ is channel width, $D$ is particle diameter, $Q$ is flow rate, $S_{0}$ is bed surface slope, $U=$ $Q / B H$ is bulk velocity, $u_{*}=\sqrt{g H S_{0}}$ is shear velocity, $R=U H / v$ is bulk Reynolds number, $\mathrm{Fr}=U / \sqrt{g H}$ is Froude number, the + superscript denotes normalization with the viscous length scale $v / u_{*}, v$ is fluid kinematic viscosity, and $g$ is acceleration due to gravity.

spherical and cylindrical lenses. Silver-coated hollow glass microspheres manufactured by Microsphere Technology Ltd were used as tracer particles (20-32 $\mu \mathrm{m}$ diameter, $0.8-1.0 \mathrm{~g} \mathrm{~cm}^{-3}$ density) at a concentration of $11 \mathrm{mg} \mathrm{l}^{-1}$, which resulted in PIV records with particle image diameters of 2.0 pixels and a particle image concentration of 0.02 particles pixel $^{-1}$. Water prisms in the optical path were used to reduce distortion and total internal reflection at the air-water interface. Images were recorded directly to a fast disk array at a rate of 32 image pairs per second per camera for a continuous duration of $120 \mathrm{~min}$ at each of the five flow configurations identified in table 1.

Analysis of the recorded images proceeds by first 'de-warping' images from each camera onto a common grid and then extracting two-component vector fields independently for each camera using cross-correlation. For this dataset we have used the cross-correlation algorithm we refer to as IDM-BL48-SincLP1.5-GD9. Here BL48 means Blackman weighted $48 \times 48$ pixel interrogation regions, SincLP1.5 is a $20 \times 20$ grid point Blackman weighted sinc kernel with low-pass factor 1.5 applied to the vector field after each iteration of the algorithm, and GD9 refers to a $9 \times 9$ pixel $(0.9 \mathrm{~mm} \times 0.9 \mathrm{~mm})$ grid spacing $(81.25 \%$ overlap between adjacent interrogation regions). The algorithm uses the iterative deformation method (IDM; Astarita \& Cardone 2005) and satisfactory convergence is reached after eight iterations. The separable transfer function $T\left(1 / \lambda_{x}, 1 / \lambda_{y}, 1 / \lambda_{z}\right)$ for the combined effects of the light 
sheet thickness and the correlation algorithm is given in figure $2(b)$, where $\lambda_{x}, \lambda_{y}$ and $\lambda_{z}$ are wavelengths in the corresponding $x, y$ and $z$ directions. The transfer function relates the measured $\left(\phi_{i i}^{m}\right)$ and actual $\left(\phi_{i i}\right)$ spectra of the velocity field according to $\phi_{i i}^{m}(\boldsymbol{k})=\phi_{i i}(\boldsymbol{k})\left\{|T|^{2}(\boldsymbol{k})\right\}$, where $\boldsymbol{k}=\left(2 \pi / \lambda_{x}, 2 \pi / \lambda_{y}, 2 \pi / \lambda_{z}\right)$ is the wavenumber vector. Figure $2(b)$ indicates that turbulence wavelengths greater than $\approx 3 \mathrm{~mm}$ are resolved by the measurement system, while smaller scales are attenuated. This measurement resolution is sufficient to identify and quantify the LSMs and the VLSMs that are of primary interest in this study. However, second-order bulk statistics are likely to be underresolved in the near-bed region, i.e. contributions from scales $<3 \mathrm{~mm}$ to turbulent stresses are suppressed to a certain degree. Three-component vector fields are reconstructed from the two-component vector fields obtained for each camera using the local view angles obtained from a camera calibration procedure. The four-camera PIV system used here permits two independent estimates of each velocity component, which we use when calculating bulk statistics, correlation functions and spectra to reduce the contribution of measurement noise (for further details see Cameron et al. 2013).

\section{Results}

\subsection{Bulk statistics}

In this section we confirm the two-dimensionality of the studied flows in the measurement area, examine the potential existence of a logarithmic scaling region in the mean velocity profile, and look for potential effects of flow relative submergence on bulk statistics up to order four. These statistics provide background information on the studied flows as bulk characteristics are likely to be associated with features of LSMs and VLSMs identified in the following sections.

Our experiments were conducted with large flow aspect ratios $(B / H \gtrsim 10)$, which according to traditional wisdom (e.g. Nezu \& Nakagawa 1993) should be sufficient to establish two-dimensional flow conditions at the flume centre, i.e. $\partial^{-} / \partial y=\bar{v}=\bar{w}=0$, where the overbar represents time averaging. With the long record length and small sampling error in this study, however, we are able to detect small-amplitude transverse fluctuations with period $2 H$ in all time-averaged velocity statistics consistent with the presence of secondary currents (e.g. Nezu \& Nakagawa 1993). In order to reduce the dataset and suppress weak transverse fluctuations, we incorporate spatial averaging over a transverse domain of width $n \times 2 \times H(n=3,2,1,1,1$ for H030, ., H120, respectively). With this averaging, denoted here with angle brackets, we can reasonably expect that far from the sidewall the relation $\partial\left\langle^{-}\right\rangle / \partial y \approx\langle\bar{v}\rangle \approx\langle\bar{w}\rangle \approx 0$ will hold. In this case the double-averaged momentum conservation equation (e.g. Nikora et al. 2007) in the streamwise direction for the flow region above the roughness tops reduces to

$$
-\rho g S_{0}=\frac{\partial}{\partial z}\left\{-\rho\langle\tilde{\bar{u}} \tilde{\bar{w}}\rangle-\rho\left\langle\overline{u^{\prime} w^{\prime}}\right\rangle+\mu \frac{\partial\langle\bar{u}\rangle}{\partial z}\right\}
$$

where $\rho$ is fluid density, $\mu$ is fluid dynamic viscosity and we decompose the $i$ th instantaneous velocity component as $u_{i}=\left\langle\bar{u}_{i}\right\rangle+\tilde{\bar{u}}_{i}+u_{i}^{\prime}$, where tilde and prime symbols represent space and time fluctuations, respectively. Away from the bed, the form-induced stress $-\rho\langle\tilde{\bar{u}} \tilde{\bar{w}}\rangle$ can be used as a measure of the contribution of secondary currents to the total momentum flux. Figure 3(c) indicates that for our experiments $\langle\tilde{\bar{u}} \tilde{\bar{w}}\rangle / u_{*}^{2}$ is quite small $(\approx-0.006$ at $z / H=0.5)$ and $\left\langle\overline{u^{\prime} w^{\prime}}\right\rangle / u_{*}^{2}$ approaches the linear trend expected for two-dimensional OCF. We therefore conclude that 

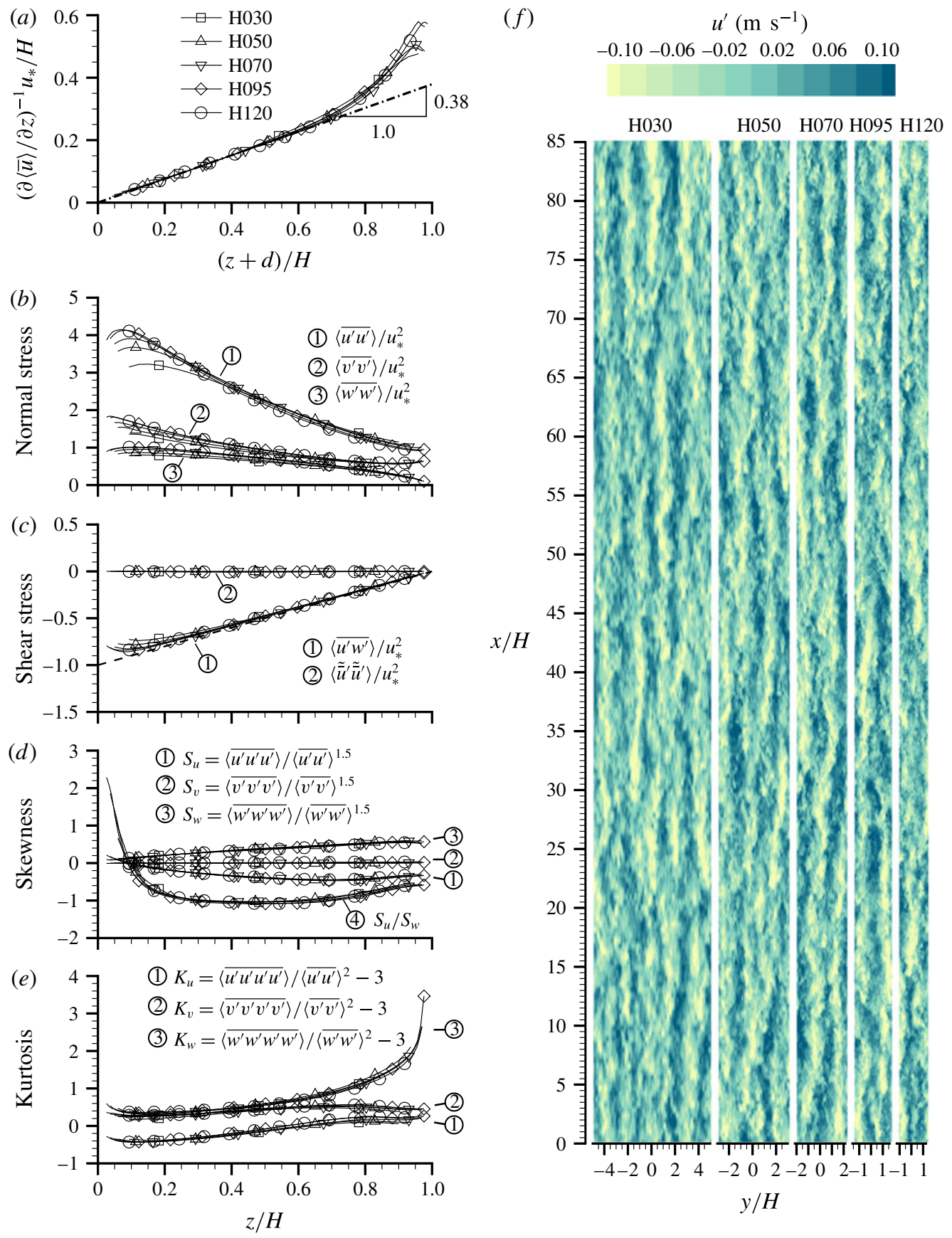

$y / H$

FIgURE 3. (Colour online) ( $a-e)$ Bulk statistics and $(f)$ distribution of 'instantaneous' velocity fluctuations at $z / H=0.5$. Symbols shown every tenth measurement point for clarity. 
secondary currents make only a minimal (essentially negligible) contribution to the total momentum flux and thus the studied flow region can safely be treated as two-dimensional.

The overlap region of wall-bounded flows where the mean velocity is expected to scale with the log of wall-normal distance remains the subject of intensive theoretical and experimental research (e.g. Adrian \& Marusic 2012). Debate has largely centred around the limits of the log region and the appropriate value of the von Kármán coefficient $(\kappa)$ and its potential variation between different flow types. The data suggest that the von Kármán coefficient is probably not universal and for smooth walls has a value of around 0.41 for pipe flow, 0.38 for boundary layers, 0.37 for closed channels (Nagib \& Chauhan 2008), and 0.41 for open channels (Nezu \& Nakagawa 1993). It should be noted, however, that experimental uncertainty for the value of $\kappa$ is still high (e.g. \pm 0.02 , Bailey et al. 2014) and its estimates will continue to be refined as new and more accurate data become available. For rough-bed flows, analysis of the $\log$ scaling region is further complicated by an additional free parameter in the equation for mean velocity, the so-called zero-plane displacement $(d)$, which sets the 'virtual' bed level for the log law. Nikora et al. (2002) proposed that conceptually the zero-plane displacement could be considered as the level that large-scale attached eddies 'feel' as the bed and, therefore, their dimensions should scale linearly with the distance from this location. The differential form of the log law incorporating the zero-plane displacement can be written as $(\partial \bar{u} / \partial z)^{-1}=\kappa(z+d) / u_{*}$ (Nikora et al. 2002), where $z=0$ corresponds to the level of the roughness tops. This 'diagnostic' form of the log law is preferable for analysing experimental data, as small deviations from the logarithmic behaviour are easy to detect. The linear relation between the inverse velocity derivative and the vertical coordinate in $(\partial \bar{u} / \partial z)^{-1}=\kappa(z+d) / u_{*}$ also provides a simple and unambiguous technique for estimation of $d$ and $\kappa$ (Nikora et al. 2002). Thus, the relation $(\partial \bar{u} / \partial z)^{-1}=\kappa(z+d) / u_{*}$ has been used in our study first to identify the existence of the logarithmic region and its bounds, and then to obtain values of $d$ and $\kappa$. Before we summarise the outcomes of our analysis, we should mention that for flows with low relative submergence (small $H / D$ ) the log layer may be disrupted by the roughness elements and appear with modified $\kappa$ (e.g. Dittrich $\&$ Koll 1997); for sufficiently small $H / D$ the log layer is not expected to exist at all (e.g. Jiménez 2004). Owing to a lack of quality experimental data for low-submergence OCF, the flow conditions required for a logarithmic layer to develop have not yet been established and thus our data may help with clarifying this matter. Figure 3(a) shows that the approximation of the measured data with the logarithmic equation is highly accurate, at least within $z / D>0.5$ and $z / H<0.5$. Zero-plane displacements, estimated using a regression fit to the inverse velocity gradient between $z / D>0.5$ and $z / H<0.4$, indicate a 'virtual' bed level to be just below the roughness tops, i.e. $d=1.8,1.8,1.6,1.1,2.0 \mathrm{~mm}$ for $\mathrm{H} 030, \ldots, \mathrm{H} 120$, respectively. No clear trend in the obtained values of the zero-plane displacement is observed, suggesting that variation in estimates of $d$ with relative submergence is probably due to experimental uncertainties. It is somewhat surprising, given the low submergence of the studied flows, to find that the present data support a logarithmic range with $\kappa=0.38$ extending to $z / H \approx 0.5$ (figure $3 a$ ), well beyond the $z / H \approx 0.15$ upper bound expected in other flow types. The extended range of the log scaling observed here suggests that the low-submergence log law may develop due to different physical mechanisms and require different theoretical justification compared to its high-submergence and smooth-bed counterparts. These differences may be further explored as new accurate data at other flow conditions and different roughness types become available. 
Second-order statistics $\left\langle\overline{u^{\prime} u^{\prime}}\right\rangle,\left\langle\overline{v^{\prime} v^{\prime}}\right\rangle$ and $\left\langle\overline{w^{\prime} w^{\prime}}\right\rangle$ (figure $3 b$ ) indicate a trend of increasing variance in the near-bed region with growing submergence. Such an effect has been noted before (e.g. Bayazit 1976; Dittrich \& Koll 1997) although the physical mechanisms behind this trend remain unclear. Velocity skewness and kurtosis (figure $3 d, e$ ) collapse onto common curves for all experiments, suggesting that these distributions might be independent of relative submergence. Skewness and kurtosis data for open channels are relatively scarce, but we find qualitative agreement between our measurements and those of Hurther \& Lemmin (2000), Nikora \& Goring (2000b) and Bigillon, Niño \& Garcia (2006). The ratio between streamwise and bed-normal velocity skewness approaches a constant value of $-1.05 \pm 0.05$ near the mid-depth of the flow (figure $3 d$ ) consistent with the assertion of Raupach (1981) of a proportional relationship between third moments.

\subsection{Large-scale streaks}

The pseudo-instantaneous velocity field (figure $3 f$ ) reconstructed from time-series records using Taylor's frozen turbulence hypothesis is composed of regions of highand low-momentum fluid alternating in the transverse direction and elongated in the streamwise direction, visually very similar to the superstructures identified by Hutchins \& Marusic (2007) in their boundary layer studies. It is not easy to directly identify length scales of the large streaks from the 'instantaneous' flow fields, as they tend to meander, fade and merge with other features without providing discernible start and end points. In the next section we will use correlation functions and spectra to extract characteristic scales and examine the potential influence of relative submergence.

\subsection{Correlation functions and spectra}

Selected components of the two-point correlation tensor,

$$
C u_{i} u_{j}\left(\Delta y, \Delta z, z_{0}\right)=\frac{\sum^{y} \sum^{t} u_{i}^{\prime}\left(y, z_{0}, t\right) u_{j}^{\prime}\left(y+\Delta y, z_{0}+\Delta z, t\right)}{\left(\sum^{y} \sum^{t}\left[u_{i}^{\prime}\left(y, z_{0}, t\right)\right]^{2}\right)^{0.5}\left(\sum^{y} \sum^{t}\left[u_{j}^{\prime}\left(y+\Delta y, z_{0}+\Delta z, t\right)\right]^{2}\right)^{0.5}},
$$

calculated assuming homogeneity in the transverse direction are plotted in figure 4 for $z_{0}=0.5 \mathrm{H}$; where $t$ is a time index, $z_{0}$ is a reference elevation, and $\Delta_{y}$ and $\Delta_{z}$ are displacement increments. The $-C_{u w}$ and $-C_{u v}$ components when plotted as vectors (as in Marusic \& Hutchins 2008) indicate that fluctuations in the streamwise velocity component are correlated with a pattern of repeating counter-rotating depth-scale cells, which may be associated with the large-scale streaks identified in figure $3(f)$. Note that we adopt vectors $\left(-C_{u w},-C_{u v}\right)$ for consistency with Marusic \& Hutchins (2008), who pointed out that such a vector map is approximately equivalent to the linear stochastic estimate where the condition vector is a negative sign $u$ event at the reference point. As it is important to show that the counter-rotating cell pattern repeats (there are four cells visible in figure 4$)$, we show vectors $\left(-C_{u w},-C_{u v}\right)$ with unit magnitudes to prevent attenuating visual effects due to a wide range of correlation values. Figure 4 shows that, with increasing submergence, the transverse separation of the cells becomes progressively smaller and the central lobe of the $C_{u u}$ correlation shrinks. The counter-rotating depth-scale cells are reminiscent of secondary current flow patterns that appear near sidewalls in open channels (e.g. Nezu \& Nakagawa 1993). The origin, dynamics and energetics of secondary currents in OCF are still 


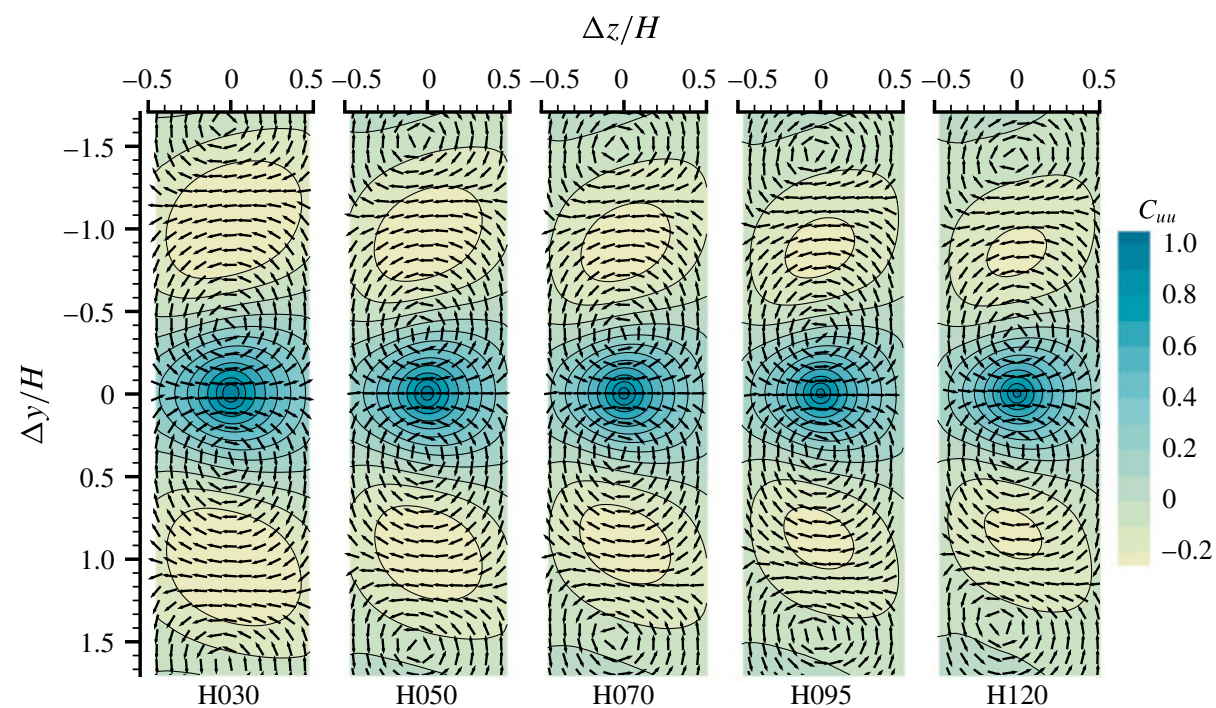

FIgURE 4. (Colour online) Two-point correlation functions $C_{u u}, C_{u w}$ and $C_{u v}$ for $z_{0} / H=$ 0.5. Note that $C_{u w}$ and $C_{u v}$ are shown as vectors $\left(-C_{u w},-C_{u v}\right)$ that have unit magnitude for a sharper identification of rotational patterns.

the subject of ongoing research and it is possible that they form due to a preferential alignment of meandering VLSMs caused by the channel sidewalls (see also Adrian \& Marusic (2012) for discussion of potential links between VLSMs and secondary currents).

To identify the prevailing scales in the flow field, we used pre-multiplied wavenumber spectra as in other LSM and VLSM studies (e.g. Kim \& Adrian 1999; Hutchins \& Marusic 2007; Monty et al. 2009). The pre-multiplied spectra for the streamwise velocity component $\left(k_{x} F_{u u}\left(k_{x}\right) / u_{*}^{2}\right.$, where $k_{x}=2 \pi / \lambda_{x}$ and $\lambda_{x}$ is streamwise wavelength) were calculated in the frequency domain and averaged over $225 \times$ $32 \mathrm{~s}$ windows with, assuming homogeneity, additional averaging over the transverse extent of the measurement domain. Taylor's frozen turbulence hypothesis was used to transform the frequency spectra to the wavenumber domain using a convection velocity equal to the local mean velocity $\langle\bar{u}\rangle$. Recognising the potential limitations of applying Taylor's hypothesis at large scales and uncertainty as to the appropriate value of the convection velocity, which may diverge from the local mean velocity in the region $z / H<0.1$ (Nikora \& Goring 2000a; Cameron \& Nikora 2008; Stewart 2014), the spectral estimates presented here may be distorted from the true wavenumber spectra. Nevertheless, our methodology is consistent with the approach used in other studies (e.g. Kim \& Adrian 1999; Hutchins \& Marusic 2007). The spectra (figure 5) reveal two characteristic length scales in the outer flow and resemble spectra measured in pipes and closed channels with smooth-wall conditions (e.g. Monty et al. 2009). Following the terminology of Kim \& Adrian (1999), we will refer to flow structures associated with the shorter of these length scales as LSMs and structures associated with the larger length scale as VLSMs. The length scales determined from spectral peaks may not directly relate to the physical length of turbulent structures due to their transverse meandering (Hutchins \& Marusic 2007) but, nevertheless, the spectral length scale provides a convenient and consistent reference to compare with other studies. Near the bed $(z / H<0.1)$ the spectral maps are dominated by a single length 

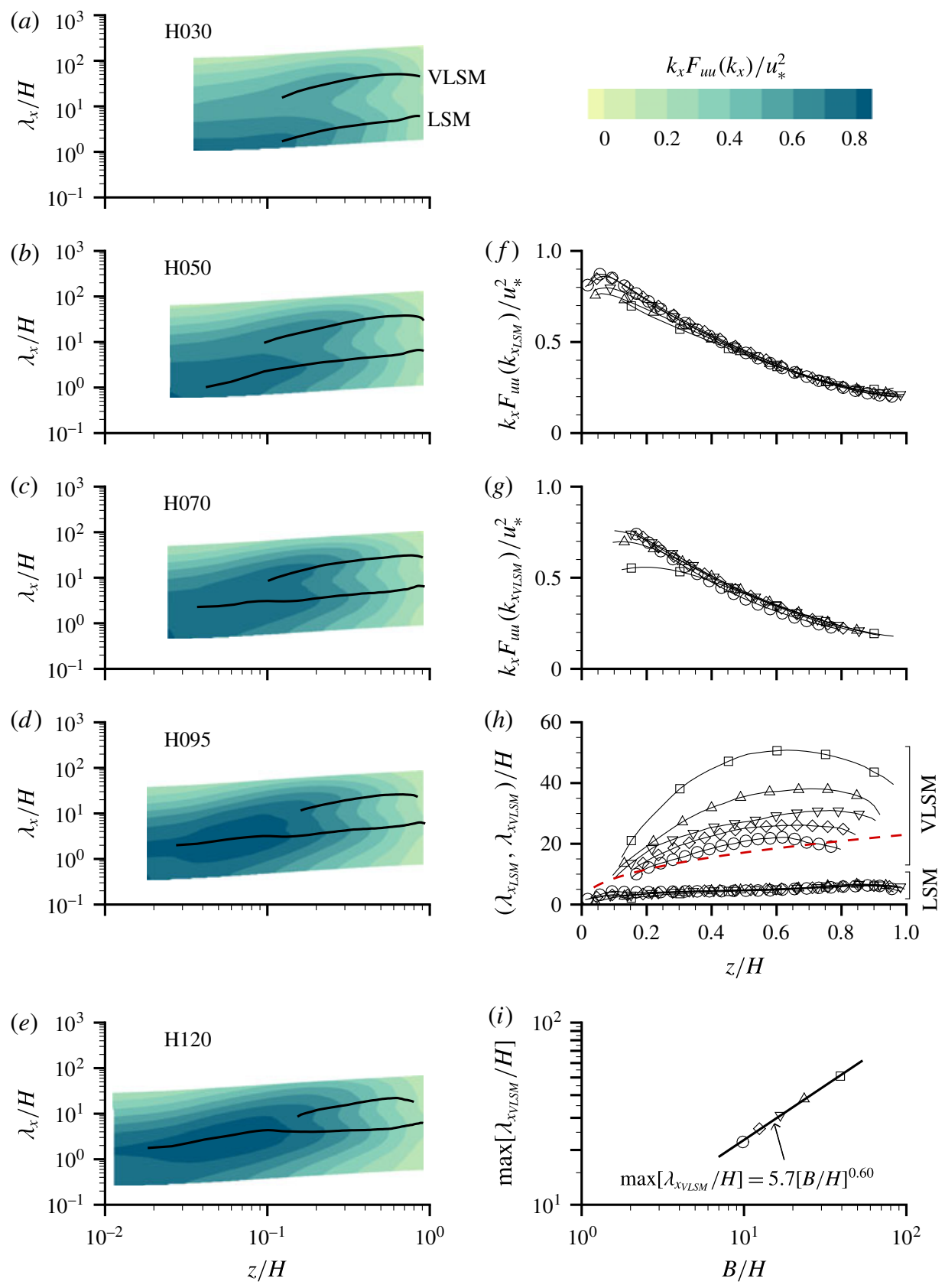

FIGURE 5. (Colour online) ( $a-e)$ Pre-multiplied spectra; amplitude of the $(f)$ LSM and $(g)$ VLSM spectral peaks; $(h)$ wavelengths of the LSM and VLSM peaks; and (i) maximum wavelength of the VLSM versus flow aspect ratio. Symbols are defined in figure 3. Dashed line in panel $(h)$ corresponds to $\lambda_{x_{V L S M}} / H=23(z / H)^{3 / 7}$ proposed by Monty et al. (2009).

scale and experiments with larger $H$ suggest a bifurcation point where the distinct LSM and VLSM scales emerge. Peak amplitudes of the pre-multiplied spectra at the LSM and VLSM wavelengths $\left(\lambda_{x_{L S M}}\right.$ and $\lambda_{x_{V L S M}}$ respectively) are approximately the 
same (figure 5f,g). Amplitude dependence on flow submergence is observed near the bed, consistent with the trend noted earlier for the bulk variance $\left\langle\overline{u^{\prime} u^{\prime}}\right\rangle$. Combining the normalised LSM and VLSM length scales for all experiments (figure $5 h$ ), it is surprising to find that, although estimates of the LSM length scale collapse onto a single curve, the estimates of VLSM length scale are stratified according to flow submergence (or equivalently to flow aspect ratio). In addition to the measured data, figure $5(h)$ also shows an empirical relationship $\lambda_{x_{V L S M}} / H=23(z / H)^{3 / 7}$ proposed in Monty et al. (2009) based on the available data for pipe and closed-channel flows. As one can see in figure 5(h), the Monty et al. (2009) relation represents a lower envelope curve that is close to the data corresponding to the highest submergence H120 (table 1).

Plotting the maximum of each $\lambda_{x_{V L S M}} / H$ distribution (figure $5 i$ ), the VLSMs appear to scale according to $\max \left[\lambda_{x_{V L S M}} / H\right]=5.7[\mathrm{~B} / \mathrm{H}]^{0.60}=75[\mathrm{H} / \mathrm{D}]^{-0.60}=3500\left[\mathrm{H}^{+}\right]^{-0.60}$, where, given that the ratios $B / D$ and $D u_{*} / v$ are constant for all experiments, all three depth normalisations $\left(B / H, H / D, H^{+}\right)$have equivalent empirical fit. Although further data are required to identify the most appropriate scaling for the VLSMs, we can speculate that $H / D$ scaling does not seem likely given that the smaller LSMs scale independently of the relative submergence (figure $5 h$ ). Reynolds-number $\left(H^{+}\right)$ effects have been assessed in other flow types and found to have minimal influence on VLSM length scale (e.g. Balakumar \& Adrian 2007) though the amplitudes of the pre-multiplied spectra have been found to exhibit Reynolds-number dependence (Hutchins \& Marusic 2007). Flow aspect ratio $(B / H)$ is perhaps the most plausible scaling given that $B$ and $\lambda_{x_{V L S M}}$ are of the same order of magnitude and keeping in mind the potential constraining effect of the flow width.

The two-dimensional pre-multiplied spectrum $k_{x} k_{y} F_{u u}\left(k_{x}, k_{y}\right) /\left\langle\overline{u^{\prime} u^{\prime}}\right\rangle$ indicates the distribution of energy across streamwise $\left(\lambda_{x}\right)$ and transverse $\left(\lambda_{y}\right)$ wavelengths. We calculate the two-dimensional spectrum in the hybrid transverse wavenumberfrequency domain and subsequently transform the frequency to equivalent streamwise wavenumber using Taylor's hypothesis. The contribution of VLSMs is readily apparent in the two-dimensional spectra (figure 6), as they concentrate energy into a narrow range of transverse scales around $\lambda_{y}=2 H$ for the shallower depth experiments $(\mathrm{H} 030, \ldots, \mathrm{H} 070)$. For the deeper flow experiments (H095, H120), the transverse wavelength of the VLSM appears to be shorter, but this may be due to the limited resolution in $k_{y}$ for these experiments (as the PIV measurement window becomes smaller in terms of $H$ ). The transverse scale of the LSM is elevation-dependent and increases along with the streamwise length scale with increasing $z$. The VLSM transverse scale in contrast appears to be constant for all $z$.

Data presented here indicate that, in rough-bed OCFs, VLSMs scale differently to LSMs in terms of both their transverse and streamwise length scales. These scaling differences suggest that VLSMs may form independently of LSMs rather than as a streamwise alignment of the smaller structures. Further experimental data are required to resolve scaling and formation mechanisms of LSMs and VLSMs in rough-bed OCFs.

\section{Conclusions}

The obtained pre-multiplied spectra in rough-bed OCF exhibit a bimodal shape in the outer flow corresponding to the presence of LSMs and VLSMs. The latter motions are also evident as large-scale streaks in instantaneous streamwise velocity fields. Two-point correlations suggest that VLSMs may be associated with repeating 


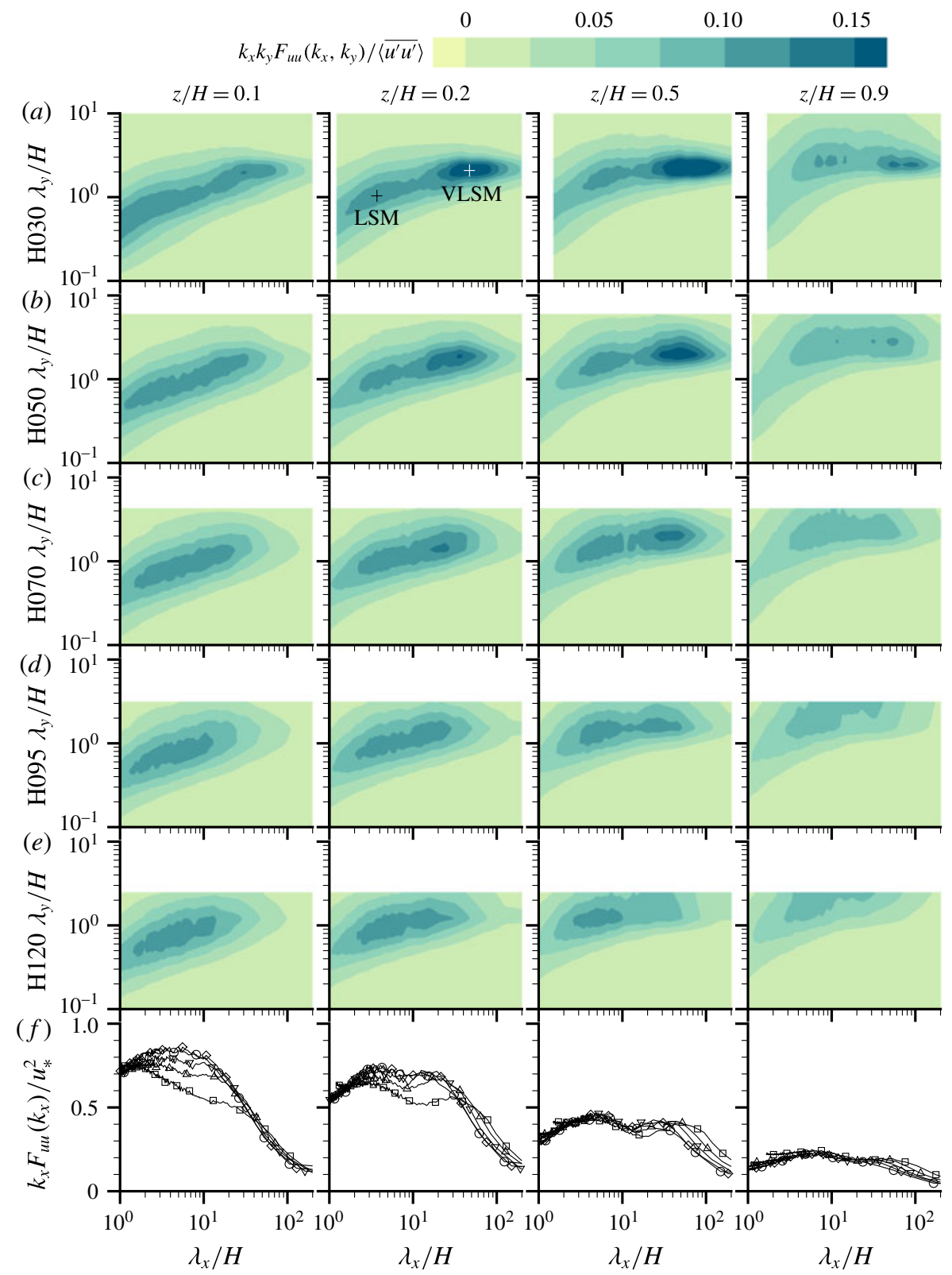

FIgURE 6. (Colour online) ( $a-e)$ Pre-multiplied two-dimensional spectra (contours) and $(f)$ pre-multiplied one-dimensional spectra. Symbols as in figure 3 . The $95 \%$ confidence interval for the one-dimensional spectra is approximately $0.94 \times$ to $1.07 \times k_{x} F_{u u}\left(k_{x}\right) / u_{*}^{2}$.

depth-scale counter-rotating cells reminiscent of and potentially related to secondary currents in the mean (time-averaged) flow. The energy contents of the two motion scales are approximately the same, although some dependence of the spectral shape and amplitudes on relative submergence is found in the near-bed region. The streamwise length scale of the LSM collapses across experiments when normalised with the flow depth. However, the depth-normalised VLSM length scale does not 
collapse. Instead, it exhibits a power-type dependence on either Reynolds number $H^{+}$, relative submergence $H / D$ or flow aspect ratio $B / H$. Since in our experiments we kept channel width, particle size and shear velocity constant, we cannot unambiguously identify which particular factor $\left(H / D, B / H\right.$ or $\left.H^{+}\right)$is responsible for the variation of the depth-normalised VLSM length scale. Further data are required to establish which of them is most appropriate and physically justifiable. At this stage we can only speculate that the true scaling is associated with the aspect ratio $B / H$. The transverse length scale of VLSMs is fixed near $2 H$ for all elevations but the transverse scale of LSMs is elevation-dependent. Differences in the scaling of LSMs and VLSMs suggest that these two motions may form independently of each other. Additionally, the obtained data support the existence of a logarithmic scaling region with $\kappa=0.38$ extending to $z / H \approx 0.5$ despite the low relative submergence of the studied flows.

Uniform open-channel flows exhibit a number of unique features differentiating them from other canonical flow types (pipes, closed channels and boundary layers): (1) unlike boundary layer thickness, which increases along the flow, OCF depth does not change; (2) corners at the intersections of the channel walls, bed and free surface of OCFs generate turbulence anisotropy that induce secondary currents (in very wide channels the secondary currents may weaken or even die out away from the channel walls); and (3) the free surface acts as a mobile moving boundary and may have significant impact on flow dynamics. Altogether, these features make OCF a special class of wall-bounded turbulent flows distinctly different from boundary layers, closed channels and pipes where the free surface is absent. The discovery of VLSMs in this flow type highlights some universality in wall-bounded flows at very large scales and may help in resolving the puzzle of VLSM origin and dynamics.

\section{Acknowledgements}

The study has been supported by two EPSRC/UK grants, 'High-resolution numerical and experimental studies of turbulence-induced sediment erosion and near-bed transport' (EP/G056404/1) and 'Bed friction in rough-bed free-surface flows: a theoretical framework, roughness regimes, and quantification' (EP/K041169/1). Discussions with I. Marusic and comments of three anonymous reviewers are greatly appreciated.

\section{REFERENCES}

AdRIAN, R. J. \& MARUSIC, I. 2012 Coherent structures in flow over hydraulic engineering surfaces. J. Hydraul Res. 50 (5), 451-464.

Astarita, T. \& CARdone, G. 2005 Analysis of interpolation schemes for image deformation methods in PIV. Exp. Fluids 38 (2), 233-243.

Bailey, S. C. C., Vallikivi, M., Hultmark, M. \& Smits, A. J. 2014 Estimating the value of von Kármán's constant in turbulent pipe flow. J. Fluid Mech. 749, 79-98.

BAlAKUMAR, B. J. \& ADRIAN, R. J. 2007 Large- and very-large-scale motions in channel and boundary-layer flows. Phil. Trans. R. Soc. Lond. A 365 (1852), 665-681.

BAYAZIT, M. 1976 Free surface flow in a channel of large relative roughness. J. Hydraul Res. 14 (2), $115-126$.

Bigillon, F., Niño, Y. \& GARCIA, M. H. 2006 Measurements of turbulence characteristics in an open-channel flow over a transitionally-rough bed using particle image velocimetry. Exp. Fluids 41 (6), 857-867.

Cameron, S., Nikora, V. \& Coleman, S. 2008 Double-averaged velocity and stress distributions for hydraulically-smooth and transitionally-rough turbulent flows. Acta Geophys. 56 (3), 642-653. 
Cameron, S. M. \& Nikora, V. I. 2008 Eddy convection velocity for smooth- and rough-bed openchannel flows: particle image velocimetry study. In Proceedings. of International Conference on Fluvial Hydraulics, River Flow 2008, Turkey, vol. 1, pp. 143-150. Kubaba Congress Department and Travel Services.

Cameron, S. M., Nikora, V. I., Albayrak, I., Miler, O., Stewart, M. \& Siniscalchi, F. 2013 Interactions between aquatic plants and turbulent flow: a field study using stereoscopic PIV. J. Fluid Mech. 732, 345-372.

DitTRich, A. \& Koll, K. 1997 Velocity field and resistance of flow over rough surfaces with large and small relative submergence. Intl J. Sedim. Res. 12 (3), 21-33.

Franca, M. J. \& Brocchini, M. 2015 Turbulence in rivers. In Rivers - Physical, Fluvial and Environmental Processes, pp. 51-78. Springer.

FRANCA, M. J. \& LEMMIN, U. 2005 Cross-section periodicity of turbulent gravel-bed river flows. In Proceedings of the 4th River, Coastal and Estuarine Morphodynamics: RCEM 2005, vol. 1, pp. 203-210. CRC Press.

Grinvald, D. I. \& NiKORA, V. I. 1988 River Turbulence. Hydrometeoizdat.

Hurther, D. \& LEMmin, U. 2000 Shear stress statistics and wall similarity analysis in turbulent boundary layers using a high-resolution 3-D ADVP. IEEE J. Ocean. Engng 25 (4), 446-457.

Hutchins, N.\& MARUSic, I. 2007 Evidence of very long meandering features in the logarithmic region of turbulent boundary layers. J. Fluid Mech. 579, 1-28.

Hwang, Y. \& Cossu, C. 2010 Self-sustained process at large scales in turbulent channel flow. Phys. Rev. Lett. 105 (4), 044505.

JimÉnEZ, J. 2004 Turbulent flows over rough walls. Annu. Rev. Fluid Mech. 36, 173-196.

KIM, K. C. \& Adrian, R. J. 1999 Very large-scale motion in the outer layer. Phys. Fluids 11 (2), $417-422$.

MARUSiC, I. \& HUtchins, N. 2008 Study of the log-layer structure in wall turbulence over a very large range of Reynolds number. Flow Turbul. Combust. 81 (1-2), 115-130.

Monty, J. P., Hutchins, N., NG, H. C. H., Marusic, I. \& Chong, M. S. 2009 A comparison of turbulent pipe, channel and boundary layer flows. J. Fluid Mech. 632, 431-442.

Nagib, H. M. \& Chauhan, K. A. 2008 Variations of von Kármán coefficient in canonical flows. Phys. Fluids 20 (10), 1518.

NeZU, I. 2005 Open-channel flow turbulence and its research prospect in the 21st century. J. Hydraul. Engng 131 (4), 229-246.

NezU, I. \& Nakagawa, H. 1993 Turbulence in Open Channel Flows. Balkema.

NiKORA, V. \& GORING, D. $2000 a$ Eddy convection velocity and Taylor's hypothesis of 'frozen' turbulence in a rough-bed open-channel flow. J. Hydrosci. Hydraul. Engng 18 (2), 75-91.

NikoRA, V. \& GoRing, D. $2000 b$ Flow turbulence over fixed and weakly mobile gravel beds. $J$. Hydraul. Engng 126 (9), 679-690.

Nikora, V., Koll, K., Mclean, S., Dittrich, A. \& Aberle, J. 2002 Zero-plane displacement for rough-bed open-channel flows. In Proceedings of International Conference on Fluvial Hydraulics, River Flow 2002, Belgium, vol. 1, pp. 83-91. Balkema.

Nikora, V., McEwan, I., Mclean, S., Coleman, S., Pokrajac, D.\& Walters, R. 2007 Doubleaveraging concept for rough-bed open-channel and overland flows: theoretical background. $J$. Hydraul. Engng 133 (8), 873-883.

RAUPACH, M. R. 1981 Conditional statistics of Reynolds stress in rough-wall and smooth-wall turbulent boundary layers. J. Fluid Mech. 108, 363-382.

Roy, A. G., Buffin-Belanger, T., Lamarre, H. \& Kirkbride, A. D. 2004 Size, shape and dynamics of large-scale turbulent flow structures in a gravel-bed river. J. Fluid Mech. 500, $1-27$.

Stewart, M. T. 2014. Turbulence structure of rough-bed open-channel flow. PhD thesis, University of Aberdeen. 